\title{
Perceived influence: The role of health professionals on patients' lifestyle behaviours
}

\author{
B.J. Hermosura ${ }^{1}$, S. Aboueid ${ }^{2}$, M. Jasinska ${ }^{3}$, C. Pouliot ${ }^{4}$, I. Bourgeault ${ }^{5}$, I. Giroux ${ }^{4}$ \\ ${ }^{1}$ Faculty of Education, University of Ottawa; ${ }^{2}$ School of Public Health and Health Systems, University of \\ Waterloo; ${ }^{3}$ Faculty of Physiotherapy, Griffith University; ${ }^{4}$ Faculty of Health Sciences, University of Ottawa; \\ ${ }^{5}$ Telfer School of Management, University of Ottawa.
}

\section{OBJECTIVE:}

To understand health professionals' (HPs) perspectives on their influence on patients' lifestyle behaviours, in particular related to diet.

\section{METHODS:}

Semi-structured interviews were conducted with 33 health professionals (family physicians: $n=7$, nurse practitioners: $n=13$, dietitians: $n=13$ ) working in multidisciplinary healthcare settings.

- All interviews were audio-recorded and transcribed verbatim.

- Thematic analysis was used by two researchers (BJH and SA).

RESULTS:

\section{Degree of influence}

HPs generally believed they had a positive influence on their patients' lifestyle behaviours, while acknowledging there are uncontrollable factors.

“...then there's other people that I feel that I don't get through to for whatever reason, maybe it's the timing, my approach, where they are at in their lives, there are factors you can't control" Participant 2, NP, NPLC

\author{
Strategies to influence lifestyle behaviours \\ Strategies used to influence their patients' lifestyle \\ behaviours included leading by example and using \\ motivational interviewing techniques.
}

"They know that most of us work out at lunchtime, they see it... we lead by example. Not only are we suggesting what to do, we do it." - Participant 14, MD, FHT

"By applying motivational interviewing techniques and making patients realize that they only need to do small changes, it makes patients want to come back" - Participant 34, RD, FHT

HPs' role in influencing patients' lifestyle behaviours Practitioners' roles included discussing barriers, educating, directing, coaching, and persuading patients.

"Managing medical and social issues can help. Sometimes eating healthy is not a priority for patients but rather housing is more important, having a stable income, and there are addiction issues that can affect them as well". - Participant 19, MD, CHC

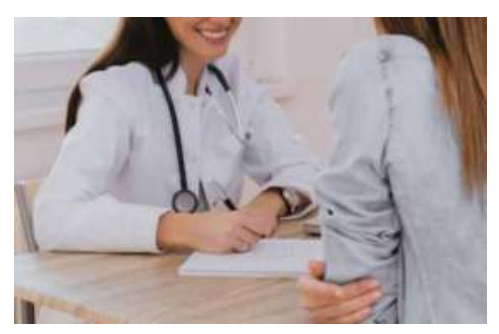

Conclusion: Most HPs perceived to have a positive influence on patients' lifestyle behaviours, which is supported by using strategies such as motivational interviewing and leading by example. Although uncontrollable factors may affect the patient's ability to change lifestyle behaviours, HPs perceived to have an important role in identifying and discussing these barriers with their patients. 